\title{
Nonlinear electrostatic oscillations in a cold magnetized electron-positron plasma
}

\author{
Prabal Singh Verma \\ CNRS/Université d'Aix-Marseille Centre saint Jérôme, case 232, F-13397 Marseille cedex 20, France \\ Tapan Chandra Adhyapak \\ Institut für Physik, Johannes Gutenberg-Universität Mainz, Staudingerweg 7-9, 55128 Mainz, Germany
}

(Dated: June 17, 2021)

\begin{abstract}
We study the spatio-temporal evolution of the nonlinear electrostatic oscillations in a cold magnetized electron-positron (e-p) plasma using both analytics and simulations. Using a perturbative method we demonstrate that the nonlinear solutions change significantly when a pure electrostatic mode is excited at the linear level instead of a mixed upper-hybrid and zero-frequency mode that is considered in a recent study. The pure electrostatic oscillations undergo phase mixing nonlinearly. However, the presence of the magnetic field significantly delays the phase-mixing compared to that observed in the corresponding unmagnetized plasma. Using 1D PIC simulations we then analyze the damping of the primary modes of the pure oscillations in detail and infer the dependence of the phase-mixing time on the magnetic field and the amplitude of the oscillations. The results are remarkably different from those found for the mixed upper-hybrid mode mentioned above. Exploiting the symmetry of the e-p plasma we then explain a generalized symmetry of our non-linear solutions. The symmetry allows us to construct a unique nonlinear solution up to the second order which does not show any signature of phase mixing but results in a nonlinear wave traveling at upper-hybrid frequency. Our investigations have relevance for laboratory/astrophysical e-p plasmas.
\end{abstract}

PACS numbers: 52.35.Mw, 52.27.Ny, 52.65.Rr

\section{INTRODUCTION}

The study of nonlinear electrostatic oscillations in a cold plasma is an interesting area of research from both theoretical and application point of view [1-7]. One of the major application of such studies is in the laser (or particle beam) induced wake-field acceleration techniques where electrostatic oscillations in plasmas are used to accelerate charged particles to high-energies in short distances 8 10. However, in the above acceleration technique, maximum acceleration can only be achieved when the amplitude of the electrostatic oscillations is kept below a critical value known as the wave-breaking amplitude [1, 3, 11]. Beyond the critical amplitude coherent oscillations break and the amplitude of the accelerating field diminishes dramatically by converting a part of the coherent energy into random kinetic energy of the particles 12]. As a result wave is no more able to accelerate particles to the desired energy. Physically, beyond the critical amplitude trajectories of neighboring oscillators taking part in the wave cross each other, and as a consequence the oscillators loose their coherent motion [1].

There is another physical process however slower but can lead to wave-breaking of arbitrarily small amplitude oscillations. This process is known as phase mixing which occurs when the frequency of the oscillations due to any physical reason (for example - inhomogeneity in the ion background 1, 13 15, relativistic mass variation effects [16 18, inhomogeneous magnetic field [19] etc.) acquires a spatial dependence. Because of the space-dependent frequency neighbouring oscillators slowly go out of phase which leads to the trajectory crossing and hence wavebreaking after certain time. Phase mixing is responsible for the flow of energy irreversibly into higher harmonics and leads to damping of the primary mode [13, 17, 20]. Thus, phase mixing is an undesirable physical process as it can significantly affect the maximum energy gain in the wake-field acceleration experiments as suggested in Ref. 18

There is considerable understanding about the phase mixing and nonlinear evolution of electrostatic oscillations in the electron-ion (e-i), and other plasmas where charged species have unequal masses [14, 15, 20, 21. Recent developments suggest electron-positron (e-p) plasma as an alternative interesting system for plasma experiments [22 25]. It is possible to produce e-p plasmas in the laboratory by laser matter interaction [26], and they also exist naturally in various astrophysical environments 27 29. The dynamics of the electrostatic oscillations in an e-p plasma differ significantly from that in the e$i$, and other plasmas referred to above, because of the equal mass of the two charged species involved. Besides, the presence of a magnetic field offers further richness to the oscillations in an e-p plasma. An external magnetic field is suggested to play a crucial role in the evolution of the upper-hybrid mode [19, 30, 31, which is a mode of electrostatic oscillations in a magnetized plasma. Although, upper-hybrid oscillations mixed with a zero frequency mode in a magnetized e-p plasma have received recent attentions 32, how a pure upper-hybrid oscillation - which lacks any other mode and therefore is easier to excite in experiments - evolves nonlinearly in space and time in a magnetized e-p plasma is still not known.

In this paper we investigate the dynamics of a pure upper-hybrid oscillation in a cold e-p plasma. Although, electrostatic oscillations in a magnetized e-p plasma has 
been studied in Ref. 32, only a mixed mode consisting of the upper-hybrid oscillations and a space-dependent zero frequency DC mode has been examined. For such mixed oscillations, the spatial dependence of the DC mode present from the beginning is directly responsible for the wave-breaking through a mechanism mentioned above. In contrast, here we choose our initial conditions such that pure upper-hybrid oscillations are set up at the linear level so that there is no cause of wave-breaking at that order. We then use a perturbative method to probe how higher order perturbations affect the pure upperhybrid mode.

We find that pure upper-hybrid oscillations in a cold ep plasma phase-mix and break at an arbitrarily small amplitudes. However, we demonstrate that, due to the presence of the magnetic field, the perturbations to the pure upper-hybrid oscillations preserve their oscillatory nature until the second order solution and hence do not grow in time. Nevertheless, higher harmonics and mixed modes are developed as the plasma acquires inhomogeneity due to the imbalance of ponderomotive forces and nonlinear Lorentz forces. We obtained the analytical solutions of all the relevant physical quantities up to the third order in a perturbative analysis. Furthermore, we explain a mathematical symmetry in the nonlinear upper-hybrid oscillations and demonstrate it up to the third order.

Our analysis of the above results imply that the presence of the magnetic field significantly suppresses the phase-mixing of the pure electrostatic mode in comparison to the fate of the electrostatic oscillations in an unmagnetized e-p plasma. For an unmagnetized e-p plasma, the use of a similar perturbative approach has established that density fluctuations in both the charged species grow in time as $\sim t^{2}$ already in the second order solution, indicating a rapid bunching of particles and hence much rapid phase mixing [20, 21, 33, 35]. Our results also reveal significant differences between the evolutions of a pure upper-hybrid oscillation and a mixed upper-hybrid mode accompanied by a space dependent DC term. In the latter case, the second order solutions grow in time and the sum of the density fluctuations acquires fast secular terms $\sim t$ already in the third order solutions. In contrast, for a pure upper-hybrid mode second order solutions do not grow in time, and although the third order solutions grow as $t \cos \omega_{h} t$ where $\omega_{h}$ is the frequency of the upper-hybrid mode, the sum of density fluctuations vanishes.

We then proceeded to perform a one dimensional particle-in-cell (1D PIC) simulation [36, first confirming a good agreement with our analytical results. Later, we estimate the phase-mixing time and its dependence on the amplitude of the oscillations and the magnetic field from our simulations. Furthermore, using the confirmed symmetry of the oscillations mentioned above, we identify an unique nonlinear solution in a cold magnetized e-p plasma which does not show any signature of phase mixing but results in a nonlinear wave propagating at the upper-hybrid frequency.
Now we proceed to present our analysis. The flow of the paper is as follows. Section II deals with the linear and nonlinear solutions of upper-hybrid oscillations in a cold e-p plasma. In section III a comparison between numerical experiment and analytical results is provided and the scalings of the phase-mixing time are estimated. Section IV describes the construction of the nonlinear solution, up to the second order, which does not show any signature of phase mixing. Section $\mathrm{V}$ contains the summary of the results obtained in this work.

\section{GOVERNING EQUATIONS AND PERTURBATION ANALYSIS}

The basic equations describing the dynamics of a cold magnetized e-p plasma are the continuity equations,

$$
\begin{aligned}
& \partial_{t} n_{e}+\nabla\left(n_{e} \mathbf{v}_{\mathbf{e}}\right)=0, \\
& \partial_{t} n_{p}+\nabla\left(n_{p} \mathbf{v}_{\mathbf{p}}\right)=0,
\end{aligned}
$$

the momentum equations,

$$
\begin{gathered}
\left(\partial_{t}+\mathbf{v}_{\mathbf{e}} \cdot \boldsymbol{\nabla}\right) \mathbf{v}_{\mathbf{e}}=-\frac{e}{m}\left[\mathbf{E}+(1 / c) \mathbf{v}_{\mathbf{e}} \times \mathbf{B}\right], \\
\left(\partial_{t}+\mathbf{v}_{\mathbf{p}} \cdot \boldsymbol{\nabla}\right) \mathbf{v}_{\mathbf{p}}=\frac{e}{m}\left[\mathbf{E}+(1 / c) \mathbf{v}_{\mathbf{p}} \times \mathbf{B}\right],
\end{gathered}
$$

and the Poisson's equation,

$$
\boldsymbol{\nabla} \cdot \mathbf{E}=4 \pi e\left(n_{p}-n_{e}\right) .
$$

Here the subscript ' $p$ ', stands for the positron and ' $e$ ', stands for the electron. The densities and velocities of both the species are denoted by $n_{\alpha}$ and $\mathbf{v}_{\alpha}$ respectively, where $\alpha$ is the corresponding subscript. The quantity $c$ is the speed of light in vacuum, $e$ is the charge of a positron (not to be confused with the subscript ' $e$ '), $\mathbf{E}$ the electric field and $\mathbf{B}$ is a homogeneous external magnetic field applied along the $z$-direction i.e. $\mathbf{B}=B_{0} \hat{z} . \quad C G S$ unit is used throughout.

Since we are interested only in the electrostatic mode, spatial variations are restricted to one direction, which we consider to be along the $x$-axis, without any loss of generality. Thus, the set of equations (1)-(5) reduces to the following equations,

$$
\begin{gathered}
\partial_{t} n_{e}+\partial_{x}\left(n_{e} v_{e x}\right)=0, \\
\partial_{t} n_{p}+\partial_{x}\left(n_{p} v_{p x}\right)=0, \\
\partial_{t} v_{e x}+v_{e x} \partial_{x} v_{e x}=-\frac{e}{m} E_{x}-\omega_{c} v_{e y}, \\
\partial_{t} v_{p x}+v_{p x} \partial_{x} v_{p x}=\frac{e}{m} E_{x}+\omega_{c} v_{p y},
\end{gathered}
$$




$$
\begin{gathered}
\partial_{t} v_{e y}+v_{e x} \partial_{x} v_{e y}=\omega_{c} v_{e x}, \\
\partial_{t} v_{p y}+v_{p x} \partial_{x} v_{p y}=-\omega_{c} v_{p x}, \\
\partial_{x} E_{x}=4 \pi e\left(n_{p}-n_{e}\right),
\end{gathered}
$$

where $\omega_{c}=e B_{0} / m c$ is the cyclotron frequency, with $m$ the common mass of both an electron and a positron. Now we proceed to adopt the perturbative analysis in order to obtain nonlinear solutions for the set of equation (6)-(12). In such analysis the general solutions can be expressed as,

$$
\begin{aligned}
& n_{e}(x, t)=n_{0}+n_{e}^{(1)}(x, t)+n_{e}^{(2)}(x, t)+n_{e}^{(3)}(x, t)+\ldots \\
& n_{p}(x, t)=n_{0}+n_{p}^{(1)}(x, t)+n_{p}^{(2)}(x, t)+n_{p}^{(3)}(x, t)+\ldots
\end{aligned}
$$

Here $n_{0}$ is the equilibrium density of both the species and the superscripts $(\beta)$ denote the order of the solutions. Different orders of the solutions are understood to be proportional to the corresponding powers of a small parameter, $\delta$, which is introduced below. Solutions for other physical quantities can similarly be written down. We now introduce the variable $n_{d}$, which is proportional to the net charge density, such that,

$$
n_{d}(x, t)=n_{p}(x, t)-n_{e}(x, t) .
$$

Since this variable will later be used in the analysis we explicitly write down its general solution as,

$$
n_{d}(x, t)=n_{d}^{(1)}(x, t)+n_{d}^{(2)}(x, t)+n_{d}^{(3)}(x, t)+\ldots
$$

\section{A. First order solution}

The set of equations $(6)-(12)$ in the first order approximation can be expressed as,

$$
\begin{gathered}
\partial_{t} n_{e}^{(1)}+n_{0} \partial_{x} v_{e x}^{(1)}=0 \\
\partial_{t} n_{p}^{(1)}+n_{0} \partial_{x} v_{p x}^{(1)}=0 \\
\partial_{t} v_{e x}^{(1)}=-\frac{e}{m} E_{x}^{(1)}-\omega_{c} v_{e y}^{(1)}, \\
\partial_{t} v_{p x}^{(1)}=\frac{e}{m} E_{x}^{(1)}+\omega_{c} v_{p y}^{(1)}, \\
\partial_{t} v_{e y}^{(1)}=\omega_{c} v_{e x}^{(1)}, \\
\partial_{t} v_{p y}^{(1)}=-\omega_{c} v_{p x}^{(1)}, \\
\partial_{x} E_{x}^{(1)}=4 \pi e\left(n_{p}^{(1)}-n_{e}^{(1)}\right)=4 \pi e n_{d}^{(1)} .
\end{gathered}
$$

Equations (16)- 19 and 22 are combined to get,

$$
\partial_{t}^{2} \delta n_{d}^{(1)}+2 \omega_{p}^{2} \delta n_{d}^{(1)}+\omega_{c} n_{0} \partial_{x}\left(v_{e y}^{(1)}+v_{p y}^{(1)}\right)=0,
$$

where, $\omega_{p}=\sqrt{4 \pi n_{0} e^{2} / m}$ is the plasma frequency of either of the species. Now equations 20 -21 and equations 16)-17) give,

$$
\partial_{t}\left(n_{0} \partial_{x}\left(v_{e y}^{(1)}+v_{p y}^{(1)}\right)=\partial_{t}\left(\omega_{c} n_{d}^{(1)}\right),\right.
$$

Integrating equation (24) w.r.t. $t$ we get,

$$
n_{0} \partial_{x}\left(v_{e y}^{(1)}+v_{p y}^{(1)}\right)=\omega_{c} n_{d}^{(1)}+C_{1} .
$$

Here the constant $C_{1}$ has to be determined from the initial conditions. In order to see pure upper-hybrid oscillations in the linear solution, initial conditions need to be chosen such that $C_{1}$ becomes zero. Otherwise, a mixed mode results in DC terms which can trigger phase mixing nonlinearly 32 . Therefore, we choose to perturb the system as follows,

$$
\begin{gathered}
n_{e}(x, 0)=n_{0}, v_{e x}(x, 0)=\frac{\delta}{2} \frac{\omega_{h}}{k} \sin (k x), v_{e y}(x, 0)=0, \\
n_{p}(x, 0)=n_{0}, v_{p x}(x, 0)=-\frac{\delta}{2} \frac{\omega_{h}}{k} \sin (k x), v_{p y}(x, 0)=0,
\end{gathered}
$$

where $\omega_{h}=\sqrt{\left(2 \omega_{p}^{2}+\omega_{c}^{2}\right)}$ and $\delta$ is a small parameter controlling the amplitudes of the perturbations. For the chosen initial conditions equation 25 reduces to,

$$
n_{0} \partial_{x}\left(v_{e y}^{(1)}+v_{p y}^{(1)}\right)=\omega_{c} n_{d}^{(1)} .
$$

From equations (27) and (26) we get,

$$
\partial_{t}^{2} n_{d}^{(1)}+\omega_{h}^{2} n_{d}^{(1)}=0
$$

Thus, the quantity $\omega_{h}$, defined earlier, is the upperhybrid frequency. Now the solution in the first order becomes,

$$
\begin{aligned}
n_{e}^{(1)} & =-\frac{n_{0} \delta}{2} \cos (k x) \sin \left(\omega_{h} t\right), \\
n_{p}^{(1)} & =\frac{n_{0} \delta}{2} \cos (k x) \sin \left(\omega_{h} t\right), \\
v_{e x}^{(1)} & =\frac{\delta}{2} \frac{\omega_{h}}{k} \sin (k x) \cos \left(\omega_{h} t\right), \\
v_{p x}^{(1)} & =-\frac{\delta}{2} \frac{\omega_{h}}{k} \sin (k x) \cos \left(\omega_{h} t\right), \\
v_{e y}^{(1)} & =\frac{\delta}{2} \frac{\omega_{c}}{k} \sin (k x) \sin \left(\omega_{h} t\right), \\
v_{p y}^{(1)} & =\frac{\delta}{2} \frac{\omega_{c}}{k} \sin (k x) \sin \left(\omega_{h} t\right), \\
E_{x}^{(1)} & =\frac{4 \pi e n_{0} \delta}{k} \sin (k x) \sin \left(\omega_{h} t\right) .
\end{aligned}
$$

From the linear solution we see that all the physical quantities oscillate coherently with the upper-hybrid frequency and there is no cause of phase mixing at the linear level. In the next subsection second order solution is obtained. 


\section{B. Second order solution}

The set of equations $(6)-(12)$ in the second order approximation can be expressed as,

$$
\begin{gathered}
\partial_{t} n_{e}^{(2)}+n_{0} \partial_{x} v_{e x}^{(2)}+\partial_{x}\left(n_{e}^{(1)} v_{e x}^{(1)}\right)=0, \\
\partial_{t} n_{p}^{(2)}+n_{0} \partial_{x} v_{p x}^{(2)}+\partial_{x}\left(n_{p}^{(1)} v_{p x}^{(1)}\right)=0, \\
\partial_{t} v_{e x}^{(2)}+v_{e x}^{(1)} \partial_{x} v_{e x}^{(1)}=-\frac{e}{m} E_{x}^{(2)}-\omega_{c} v_{e y}^{(2)}, \\
\partial_{t} v_{p x}^{(2)}+v_{p x}^{(1)} \partial_{x} v_{p x}^{(1)}=\frac{e}{m} E_{x}^{(2)}+\omega_{c} v_{p y}^{(2)}, \\
\partial_{t} v_{e y}^{(2)}+v_{e x}^{(1)} \partial_{x} v_{e y}^{(1)}=\omega_{c} v_{e x}^{(2)}, \\
\partial_{t} v_{p y}^{(2)}+v_{p x}^{(1)} \partial_{x} v_{p y}^{(1)}=-\omega_{c} v_{p x}^{(2)}, \\
\partial_{x} E_{x}^{(2)}=4 \pi e\left(n_{p}^{(2)}-n_{e}^{(2)}\right)=4 \pi e n_{d}^{(2)},
\end{gathered}
$$

From the above equations one can easily deduce that,

$$
n_{d}^{(2)}=0, \quad \text { i.e. } \quad n_{p}^{(2)}=n_{e}^{(2)} .
$$

Similar results have also been observed in the absence of a magnetic field, for example by substituting $\Delta=1$ in Ref. 21. Now using equation (42) back in equations (35)-(41) we obtain,

$$
E_{x}^{(2)}=0, \quad v_{e x}^{(2)}=v_{p x}^{(2)}, \quad v_{e y}^{(2)}=-v_{p y}^{(2)} .
$$

So, we observe a symmetry in the nonlinear solution for the pure upper-hybrid oscillations set up in the first order solution. Moreover, since the nonlinear electric field $E_{x}^{(2)}$, which is responsible for the plasma frequency contribution in the second order solution, vanishes selfconsistently, upper-hybrid mode reduces to cyclotron mode in the dynamical equation. Now from equations (35)- 43 we write down the second order solution as follows,

$$
\begin{aligned}
n_{e}^{(2)}= & \frac{n_{0} \delta^{2} \omega_{h}^{2}}{\omega_{c}^{2}-4 \omega_{h}^{2}} \cos (2 k x)\left[\frac{\omega_{p}^{2}}{\omega_{c}^{2}} \cos \left(\omega_{c} t\right)\right. \\
& \left.+\frac{3}{8} \cos \left(2 \omega_{h} t\right)\right]+\frac{n_{0} \delta^{2} \omega_{h}^{2}}{8 \omega_{c}^{2}} \cos (2 k x), \\
v_{e x}^{(2)}= & \frac{\delta^{2} \omega_{h}^{2} \omega_{p}^{2}}{2 k \omega_{c}\left(\omega_{c}^{2}-4 \omega_{h}^{2}\right)} \sin (2 k x) \sin \left(\omega_{c} t\right) \\
& +\frac{\delta^{2} \omega_{h}\left(\omega_{c}^{2}+2 \omega_{h}^{2}\right)}{16 k\left(\omega_{c}^{2}-4 \omega_{h}^{2}\right)} \sin (2 k x) \sin \left(2 \omega_{h} t\right), \\
v_{e y}^{(2)}= & -\frac{\delta^{2} \omega_{c} \omega_{h}^{2}}{2 k\left(\omega_{c}^{2}-4 \omega_{h}^{2}\right)} \sin (2 k x)\left[\frac{\omega_{p}^{2}}{\omega_{c}^{2}} \cos \left(\omega_{c} t\right)\right. \\
& \left.+\frac{3}{8} \cos \left(2 \omega_{h} t\right)\right]-\frac{\delta^{2} \omega_{h}^{2}}{16 k \omega_{c}} \sin (2 k x) .
\end{aligned}
$$

Eqs. 42- 46 provide the second order solution for all the physical quantities.

We observe that the pure upper-hybrid oscillations in the linear level develops into a mixed mode due to the presence of the slow DC terms and the cyclotron mode $\omega_{c}$. We also observe the generation of the second harmonics in space and in the upper-hybrid frequency $\omega_{h}$. Remarkably, however, there is no time dependent amplitudes in the second order solutions and hence the perturbations to the pure upper-hybrid oscillations are oscillatory in nature and do not grow in time up to the second order. This is in contrast to the non-linear evolution of the mixed upper-hybrid oscillations studied in Ref. 32 . In the mixed mode study, the zero frequency mode interacts nonlinearly with upper-hybrid mode and leads to the generation of terms growing in time $\sim t \sin \left(\omega_{h} t\right)$ and $\sim t \cos \left(\omega_{h} t\right)$ in the second order solutions.

We note that the nonlinear generation of a DC term in the second order solution can also be seen in the case of upper-hybrid oscillations in a cold electron-ion plasma when ions are considered to be infinitely massive and external magnetic field is homogeneous. This can be seen from a Taylor expansion of the exact nonlinear solution considering initial amplitude to be small 2. However, the DC term gets canceled self-consistently after one complete period, therefore frequency of the system does not acquire any spatial dependence. As a result, coherent oscillations are maintained indefinitely at the upper-hybrid frequency in such plasmas. On the contrary, in the present study the nonlinear generation of the cyclotron mode $\omega_{c}$ which is not a harmonic of upperhybrid mode $\omega_{h}$ allows the plasma to acquire space dependent frequency due to the presence of two incommensurate time scales. Therefore, nonlinear generation of the cyclotron mode in the second order solution is responsible for the two simultaneous effects. It is opposing the generation of secular terms $(\sim t),\left(\sim t^{2}\right)$, as explained below, but making the frequency of the system spacedependent. Thus, though we initiate pure upper-hybrid oscillations in a cold homogeneous e-p plasma, it nonlinearly acquires inhomogeneity in space which in turn introduces a spatial dependency in the frequency. This suggests that upper-hybrid oscillations in an e-p plasma will always phase mix and break at arbitrarily small amplitude.

The absence of the time dependent amplitudes and the nonlinear generation of the DC terms in the second order solutions can be understood as follows. Since, $E_{x}^{(2)}=0$, when the magnetic field also zero there is nothing on the r.h.s. of (37) to balance the effect of the ponderomotive forces, represented by $v_{p x}^{(1)} \partial_{x} v_{p x}^{(1)}$. As a result $v_{e x}^{(2)}$ acquires a secular term $\sim t$ which in turn introduces faster terms $\sim t^{2}$ in the electron density in equation (35) 21, 35. However, in the presence of a magnetic field Eq. (37) is coupled to the Eq. (46) through $v_{p y}^{(2)}$. In that case, the slow component of the Lorentz force [see equation [46] ] balances the ponderomotive forces and the fast 
component allows the plasma species to oscillate at cyclotron frequency $\omega_{c}$. As a result a mixture of cyclotron and upper-hybrid modes appears in equation (45) but no term that grows in time is generated. Next, the DC term in 46 is originated through an indirect effect of the ponderomotive forces which becomes clear when one writes down a second order dynamical equation for $v_{e y}^{(2)}$. The DC term in $v_{e y}^{(2)}$ in turn makes the density inhomogeneous nonlinearly as can be seen from the following relation obtained from combining Eqs. 44) and (46):

$$
\partial_{x} v_{e y}^{(2)}=-\omega_{c} n_{e}^{(2)}
$$

Thus, the indirect effect of the ponderomotive forces and self-consistently generated cyclotron mode make the plasma inhomogeneous nonlinearly in the second order solution. Physically, ponderomotive force is trying to accelerate the charged particles so as to increase their bunching in time, however the Lorentz force opposes the acceleration by changing the trajectory of the charged particles. As a result particle bunching gets slower in the magnetized plasma.

We note that by taking the limit $\omega_{c} \rightarrow 0$ in Eqs. 45 and (44) we can recover the secular terms $\left(\sim t\right.$ and $\sim t^{2}$ respectively) in both the equations. Thus, our results are consistent with the previous studies [21, 35]. In the next subsection we proceed to obtain the third order solution and provide an explanation deciphering the observed symmetry in the solutions.

\section{Third order solution}

The set of equations $6-12$ in the third order approximation can be expressed as,

$$
\begin{gathered}
\partial_{t} n_{e}^{(3)}+n_{0} \partial_{x} v_{e x}^{(3)}+\partial_{x}\left(n_{e}^{(1)} v_{e x}^{(2)}+n_{e}^{(2)} v_{e x}^{(1)}\right)=0 \\
\partial_{t} n_{p}^{(3)}+n_{0} \partial_{x} v_{p x}^{(3)}+\partial_{x}\left(n_{p}^{(1)} v_{p x}^{(2)}+n_{p}^{(2)} v_{p x}^{(1)}\right)=0 \\
\partial_{t} v_{e x}^{(2)}+\partial_{x}\left(v_{e x}^{(1)} v_{e x}^{(2)}\right)=-\frac{e}{m} E_{x}^{(3)}-\omega_{c} v_{e y}^{(3)}, \\
\partial_{t} v_{p x}^{(2)}+\partial_{x}\left(v_{p x}^{(1)} v_{p x}^{(2)}\right)=\frac{e}{m} E_{x}^{(3)}+\omega_{c} v_{p y}^{(3)}, \\
\partial_{t} v_{e y}^{(3)}+v_{e x}^{(1)} \partial_{x} v_{e y}^{(2)}+v_{e x}^{(2)} \partial_{x} v_{e y}^{(1)}=\omega_{c} v_{e x}^{(3)}, \\
\partial_{t} v_{p y}^{(3)}+v_{p x}^{(1)} \partial_{x} v_{p y}^{(2)}+v_{p x}^{(2)} \partial_{x} v_{p y}^{(1)}=-\omega_{c} v_{p x}^{(3)}, \\
\partial_{x} E_{x}^{(3)}=4 \pi e\left(n_{p}^{(3)}-n_{e}^{(3)}\right)=4 \pi e n_{d}^{(3)}
\end{gathered}
$$

From the above equations we obtain the following symmetry in the third order solutions:

$$
v_{e x}^{(3)}=-v_{p x}^{(3)}, \quad v_{e y}^{(3)}=v_{p y}^{(3)}, \quad n_{e}^{(3)}=-n_{p}^{(3)} .
$$

The above symmetry is observed to be complementary to that of the second order solutions [Eqs. 42 and (43)]. The symmetry can be extended to any order as:

$$
\begin{aligned}
& n_{e}^{(r)}=(-1)^{r} n_{p}^{(r)}, \quad v_{e x}^{(r)}=(-1)^{r} v_{p x}^{(r)}, \\
& v_{e y}^{(r)}=(-1)^{r+1} v_{p y}^{(r)},
\end{aligned}
$$

where ' $r$ ' is the order of the solution. The symmetry in the solutions results from the underlying symmetry of the dynamical Eqs. (6)-(12). The symmetry of the dynamical equations in turn is related to the similarity of an electron and a positron which differ only in the sign of their charges. Thus, at any instant if we replace each electron with a positron and vice versa, $n_{e} \rightarrow n_{p}^{f}$ and $n_{p} \rightarrow n_{e}^{f}$, where the subscript ' $f$ ' refers to the values obtained after the operation. But in this case the form of the equations are preserved only if $v_{e x} \rightarrow v_{p x}^{f}, v_{p x} \rightarrow$ $v_{e x}^{f}, v_{e y} \rightarrow-v_{p y}^{f}, v_{p y} \rightarrow-v_{e y}^{f}$. Thus the quantities, $\left(n_{p}+n_{e}\right),\left(v_{p x}+v_{e x}\right)$ and $\left(v_{p y}-v_{e y}\right)$ preserve their values under the operation,

$$
\begin{gathered}
n_{p}+n_{e}=n_{p}^{f}+n_{e}^{f}, \\
v_{p x}+v_{e x}=v_{p x}^{f}+v_{e x}^{f}, \\
v_{p y}-v_{e y}=v_{p y}^{f}-v_{e y}^{f},
\end{gathered}
$$

whereas the quantities, $\left(n_{p}-n_{e}\right),\left(v_{p x}-v_{e x}\right)$ and $\left(v_{p y}+\right.$ $\left.v_{e y}\right)$ change their signs,

$$
\begin{gathered}
n_{p}-n_{e}=-\left(n_{p}^{f}-n_{e}^{f}\right), \\
v_{p x}-v_{e x}=-\left(v_{p x}^{f}-v_{e x}^{f}\right), \\
v_{p y}+v_{e y}=-\left(v_{p y}^{f}+v_{e y}^{f}\right) .
\end{gathered}
$$

From the initial conditions used, we see that $\delta \rightarrow-\delta$ under the exchanged operation performed above. Thus, in an expansion in powers of $\delta$ the quantities $\left(n_{p}+n_{e}\right),\left(v_{p x}+\right.$ $\left.v_{e x}\right)$ and $\left(v_{p y}-v_{e y}\right)$ should have only even powers of $\delta$ and the quantities, $\left(n_{p}-n_{e}\right),\left(v_{p x}-v_{e x}\right)$ and $\left(v_{p y}+v_{e y}\right)$ possess only odd powers. The symmetries in Eq. 56 then follow immediately from the perturbation expansion used for each quantity (see, for example, Eqs. 113) and (14), which are expansions in powers of the small parameter $\delta$ of our problem.

Notably, an immediate consequence of the above symmetry is that the sum of the density fluctuations $\left(n_{e}^{(3)}+\right.$ $\left.n_{p}^{(3)}\right)$ in the third order vanishes self-consistently. This is in contrast to the situation of the mixed mode studied in Ref. 32 where $\left(n_{e}^{(3)}+n_{p}^{(3)}\right)$ is found to be $\propto t$. The third order solutions obtained by us have rather cumbersome forms. To facilitate our discussion below we provide the expressions for $n_{d}^{(3)}$ and $E_{x}^{(3)}$ in the Appendix. Note that although the third order solutions contains terms proportional to $t \cos \omega_{h} t$ growing in time, there is no fast secular term(s) proportional to $\sim t$ or $t^{2}$ until the third order. This makes a proper estimation 20, of the phase mixing time analytically difficult. Therefore, we choose to estimate the scaling of phase mixing numerically using 1D PIC simulation. In the next section, we first make a 


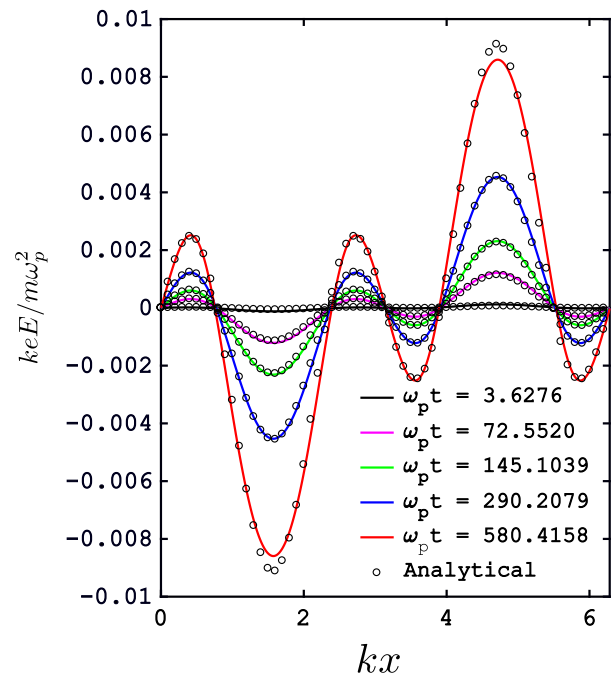

FIG. 1. Comparison between the numerical and analytical results. The normalized electric field $k e E /\left(m \omega_{p}^{2}\right)$ plotted as a function of the normalized position $k x$ where $k$ is the wave number of the longest (fundamental) mode. Solid lines are obtained from the simulation and the empty circles represent the analytical results. Different plots correspond to various time $t$ which are integral multiple of the upper-hybrid period.

comparison between the analytical results and our PIC simulation. We then proceed to investigate the evolution of the pure upper-hybrid oscillations and estimate the phase mixing time from our simulations.

\section{1D PIC SIMULATION}

In order to provide a comparison with our analytical results we carry out 1-D particle-in-cell (PIC) simulation [36] with periodic boundary conditions. We start our simulations with the same initial conditions that we used for our analysis above. Our simulation parameters are as follows: total number of both kind of particles $\mathrm{N} \sim$ $4 \times 10^{4}$, number of grid points NG $\sim 4 \times 10^{3}$, time step $\Delta t$ $\sim \pi / 50$. Normalization is as follows. $x \rightarrow k x, t \rightarrow \omega_{p} t$, $n_{e} \rightarrow n_{e} / n_{0}, v_{e} \rightarrow v_{e} /\left(\omega_{p} k^{-1}\right)$ and $E \rightarrow k e E /\left(m \omega_{p}^{2}\right)$, where $\omega_{p}$ is the plasma frequency of either of the species and $k$ is the wave number of the longest (fundamental) mode.

In the first numerical experiment maximum amplitude of the electric field $k e E /\left(m \omega_{p}^{2}\right)=\delta$ and amplitude of the external magnetic field $e B_{0} /\left(m c \omega_{p}\right)=\omega_{c} / \omega_{p}$ are chosen to be 0.04 and 1 , respectively. In Fig. 1 we show the space-time evolution of the electric field over several upper-hybrid periods where solid lines are the results from the PIC simulation and circles are the results from the nonlinear perturbative analysis up to the third order. Here, we choose to compare the results at times which are integral multiple of the upper-hybrid period $T_{h}$ such that the contribution of the first order electric-field vanishes. On the other hand, the second order electric field is always zero as we have shown in the second order solutions. Thus, it is the third order electric field $E_{x}^{(3)}$ which is playing the main role in Fig. 1. We find that the amplitude of the electric field, which should remain zero in the case of the pure oscillations, instead increases with time. Furthermore, we clearly see that there is a good agreement between the analytical and numerical results up to 80 upper-hybrid periods. However, the results start to deviate from 160 upper-hybrid periods, indicating that the effect of the higher order solutions begin to be significant at this point and beyond.

The above observation also indicates that the energy is flowing irreversibly into higher harmonics thereby damping the primary mode - a signature of phase mixing [13]. Since it is difficult to follow the trajectory crossing [1, we define the phase mixing time as the time when the amplitude of the primary mode in any physical quantity drops below $1 / e$ of its initial value. To investigate the damping and phase-mixing for varying $\delta$ and $e B_{0} /\left(m c \omega_{p}\right)$, we observe the time evolution of the primary mode $(k=1)$ of the electrostatic potential, $\phi$, which is measured in our simulations as a primary quantity from which the electric field is derived. In Fig. 2 we present four typical time evolution of the primary mode of $\phi$ corresponding to $e B_{0} /\left(m c \omega_{p}\right)=0,0.3,0.55$ and 0.75 respectively, for a fixed $\delta=0.001$. The plots show clear evidence of the damping of the primary mode after a certain time that varies for each of these cases.

We can understand the physical reason for the damping of the primary mode triggered by the phase-mixing as follows. In the absence of an external magnetic field ponderomotive forces create bunching of the charged particles leading to inhomogeneity and hence phase-mixing very quickly [see Fig2 (a)]. However, when we increase $B_{0}$ while keeping the maximum amplitude of the electric field $\delta$ constant, we actually increase the Lorentz force but keep the ponderomotive force $\sim \delta^{2}$ same. The Lorentz force opposes the ponderomotive force. Therefore, as we increase $B_{0}$ the bunching of the particles is increasingly weakened and hence the process of phasemixing gets progressively delayed. We can infer the resultant increase in the phase-mixing time as a function of $B_{0}$ from Fig. 2 where the damping of the primary mode of $\phi$ is observed to be progressively delayed from panels (a) to (d).

The inspection of the damped modes provides an estimation of the phase-mixing time, $t_{\mathrm{mix}}$, according to the definition mentioned above. In Fig. 33 (a) we show the logarithmic plots of $\omega_{p} t_{\text {mix }}$ as a function of $\delta$ for the two fixed values of $e B_{0} /\left(m c \omega_{p}\right)=0.45$ and 0.1 (denoted by the red and blue points) respectively. A linear least square fit to these plots (solid lines) reveals that $\omega_{p} t_{\text {mix }} \propto \delta^{-0.86}$ for $e B_{0} /\left(m c \omega_{p}\right)=0.45$ and $\propto \delta^{-0.81}$ for $e B_{0} /\left(m c \omega_{p}\right)=0.1$. So, the scaling of the phase-mixing time as a function of $\delta$ suggested by our simulations is approximately $\delta^{-0.83}$. We note that the scaling is different from that estimated for the mixed upper-hybrid mode for which $\omega_{p} t_{\text {mix }} \propto \delta^{-3}[32$. 
(a)

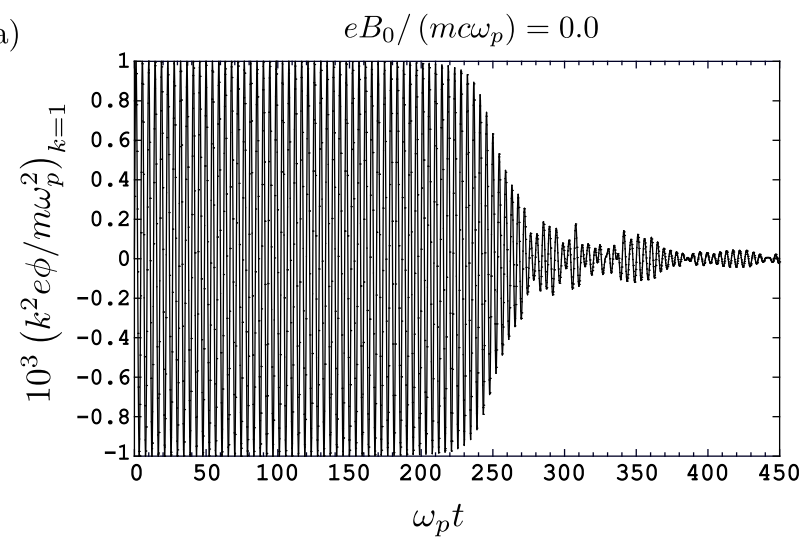

(c)

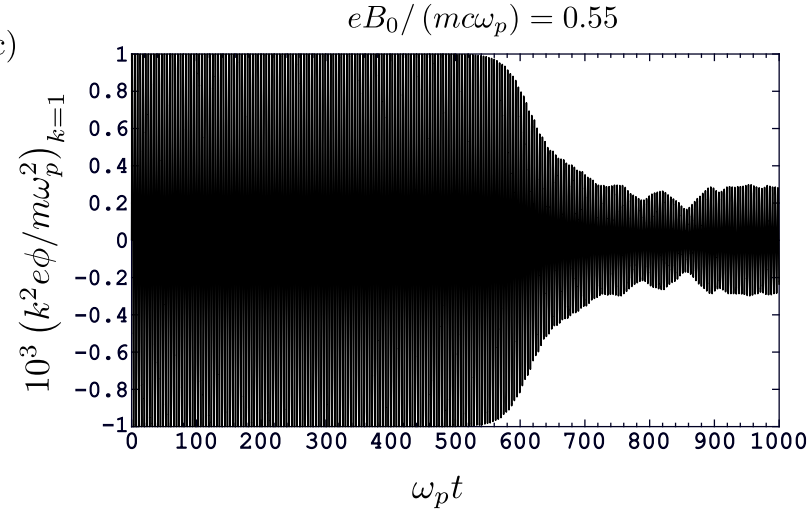

(b)

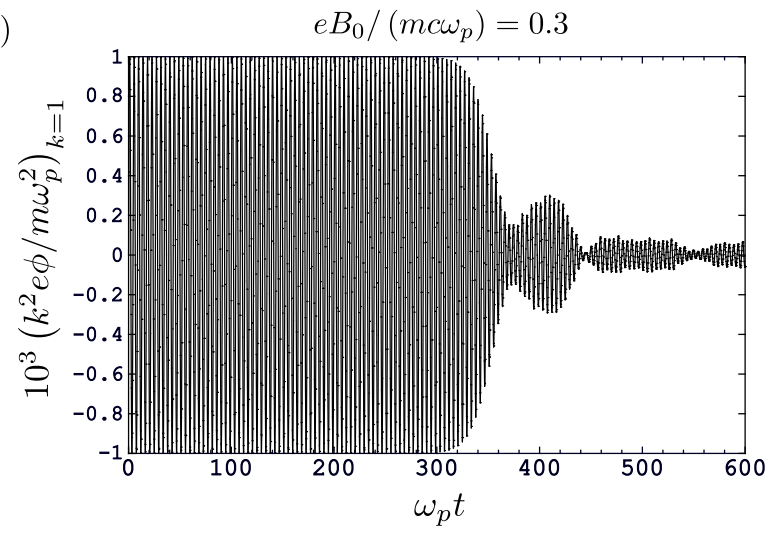

(d)

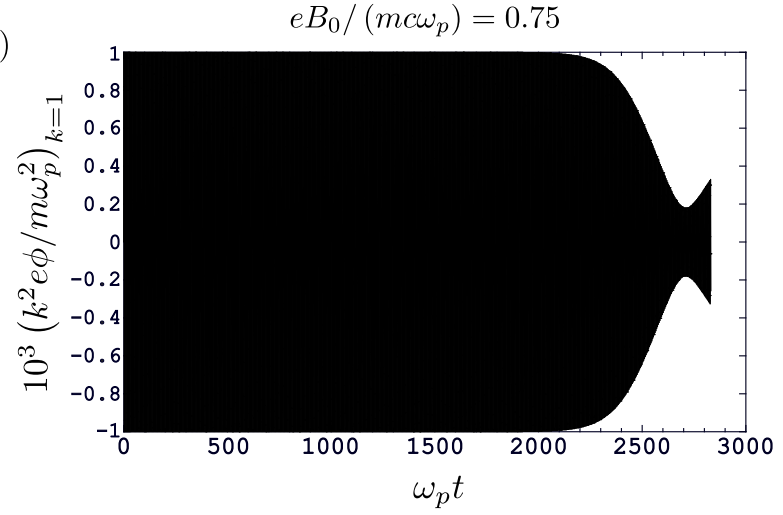

FIG. 2. Time evolution of the primary mode $(k=1)$ of the normalized electrostatic potential $k^{2} e \phi / m \omega_{p}^{2}$ with the normalized external magnetic field $e B_{0} /\left(m c \omega_{p}\right)$ set equal to (a) 0.0 , (b) 0.3 , (c) 0.55 and (d) 0.75 . In all the plots $\delta=0.001$.

The dependence of the phase-mixing time on $B_{0}$ is found to be more complex. Fig. 3 (b) shows the linear plot of $\omega_{p} t_{\mathrm{mix}}$ as a function of $e B_{0} /\left(m c \omega_{p}\right)$ for the fixed value $\delta=0.001$. We find that when $e B_{0} /\left(m c \omega_{p}\right)=0$, which is the case of unmagnetized e-p plasma, phasemixing time is $\sim 200$. For non-zero $e B_{0} /\left(m c \omega_{p}\right)$ in the range of $0-0.2$, the phase-mixing time does not change significantly. Upon further increase of $B_{0}$ a steep increase of the phase-mixing time is noticed, so that $\omega_{p} t_{\text {mix }} \sim$ 2500 at $e B_{0} /\left(m c \omega_{p}\right)=0.75$. For the chosen range of $e B_{0} /\left(m c \omega_{p}\right)$ in Fig. 3 (b), $\omega_{p} t_{\text {mix }}$ is not found to follow a single power law. This again is in contrast to the analysis provided for the mixed-upper hybrid mode [32].

In the next section we demonstrate the existence of an unique nonlinear solution which does not show any signature of phase mixing in a magnetized e-p plasma.

\section{NONLINEAR UPPER-HYBRID WAVES}

In this section, we construct a nonlinear solution for a magnetized e-p plasma which does not exhibit any phase mixing. In order to do that we choose the following initial conditions,

$$
\begin{aligned}
n_{e}^{(1)}(x, 0) & =n_{0} \frac{\delta}{2} \cos (k x), \quad n_{p}^{(1)}(x, 0)=-n_{0} \frac{\delta}{2} \cos (k x), \\
v_{e y}^{(1)}(x, 0) & =\frac{\delta}{2} \frac{\omega_{c}}{k} \sin (k x), \quad v_{p y}^{(1)}(x, 0)=\frac{\delta}{2} \frac{\omega_{c}}{k} \sin (k x), \\
v_{e x}^{(1)}(x, 0) & =-\frac{\delta}{2} \frac{\omega_{h}}{k} \sin (k x), \quad v_{p x}^{(1)}(x, 0)=\frac{\delta}{2} \frac{\omega_{h}}{k} \sin (k x) .
\end{aligned}
$$

These initial conditions lead to the following first order solutions,

$$
\begin{aligned}
n_{e}^{(1)} & =-\frac{n_{0} \delta}{2} \cos \left(k x-\omega_{h} t\right), \\
n_{p}^{(1)} & =\frac{n_{0} \delta}{2} \cos \left(k x-\omega_{h} t\right), \\
v_{e x}^{(1)} & =-\frac{\delta}{2} \frac{\omega_{h}}{k} \cos \left(k x-\omega_{h} t\right), \\
v_{p x}^{(1)} & =\frac{\delta}{2} \frac{\omega_{h}}{k} \cos \left(k x-\omega_{h} t\right), \\
v_{e y}^{(1)} & =\frac{\delta}{2} \frac{\omega_{c}}{k} \sin \left(k x-\omega_{h} t\right), \\
v_{p y}^{(1)} & =\frac{\delta}{2} \frac{\omega_{c}}{k} \sin \left(k x-\omega_{h} t\right), \\
E_{x}^{(1)} & =\delta \frac{m \omega_{p}^{2}}{k e} \sin \left(k x-\omega_{h} t\right) .
\end{aligned}
$$



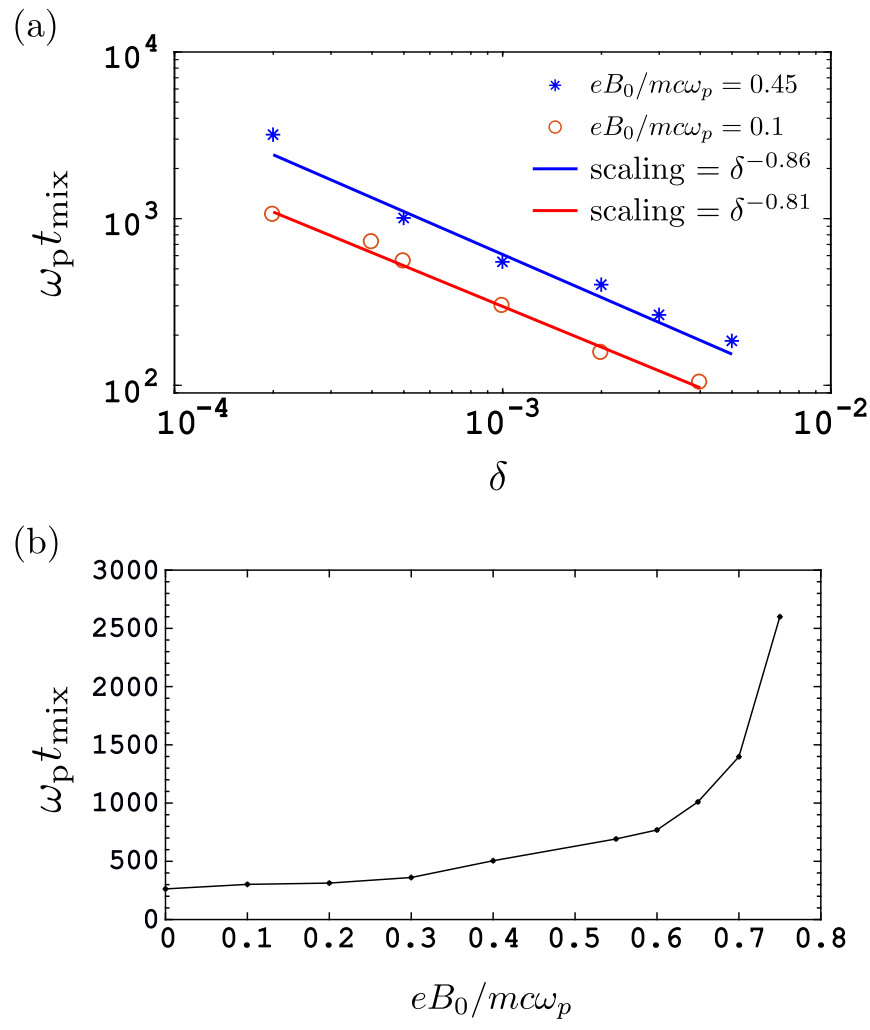

FIG. 3. (a) Logarithmic plots of the phase-mixing time versus the maximum amplitude of the electric field $\delta$. The red ('o') and blue ('*') points represent the phase mixing time estimated for $e B_{0} /\left(m c \omega_{p}\right)=0.45$ and 0.1 , respectively. The solid lines are the linear least square fits to the respective plots corresponding to the scaling $\delta^{-0.81}$ (for $e B_{0} /\left(m c \omega_{p}\right)=0.45$ ) and $\delta^{-0.86}$ (for $e B_{0} /\left(m c \omega_{p}\right)=0.1$ ) respectively. (b) Linear plots of phase-mixing time versus $e B_{0} /\left(m c \omega_{p}\right)$ estimated for $\delta=0.001$.

The above equations correspond to a pure traveling wave solution in the first order. Now in the second order we choose our initial conditions such that $n_{d}^{(2)}$ becomes zero. As a result we obtain the following relations,

$$
n_{e}^{(2)}=n_{p}^{(2)}, E_{x}^{(2)}=0, v_{e x}^{(2)}=v_{p x}^{(2)}, v_{e y}^{(2)}=-v_{p y}^{(2)} .
$$

Thus, the symmetry recognized in Sects. II B and IIC [see Eq. [56]] is also valid for the present mode. The symmetry observation for the present mode can also be inferred from the arguments similar to those presented in Sect. IIC. Using the relations in Eq. 67) we obtain all the second order quantities from the following solutions,

$$
\begin{aligned}
& n_{e}^{(2)}=-\frac{3 n_{0} \delta^{2} \omega_{h}^{2}}{4\left(\omega_{c}^{2}-4 \omega_{h}^{2}\right)} \cos \left(2 k x-2 \omega_{h} t\right), \\
& v_{e x}^{(2)}=-\frac{\delta^{2}}{8} \frac{\omega_{h}}{k} \frac{\left(\omega_{c}^{2}+2 \omega_{h}^{2}\right)}{\left(\omega_{c}^{2}-4 \omega_{h}^{2}\right)} \cos \left(2 k x-2 \omega_{h} t\right), \\
& v_{e y}^{(2)}=\frac{3 \delta^{2} \omega_{h}^{2} \omega_{c}}{8 k\left(\omega_{c}^{2}-4 \omega_{h}^{2}\right)} \sin \left(2 k x-2 \omega_{h} t\right),
\end{aligned}
$$

As in the first order, the second order solution too exhibit a traveling wave solution without any sign of phase- mixing. We thus expect that the full nonlinear solution of the mode will also be oscillatory in space and time because the ponderomotive force, a responsible candidate for phase-mixing, is zero for wave-like solutions. Thus we demonstrate that it is possible to have a oscillatory upper-hybrid mode in the e-p plasma.

\section{SUMMARY AND DISCUSSION}

We have combined analytical approach and simulations to investigate the nonlinear evolution of pure upperhybrid oscillations in a cold magnetized e-p plasma. In our analytical approach we have used a perturbative analysis where the relevant physical quantities are expanded in terms of the powers of the small parameter $\delta$ which corresponds to the maximum amplitude of the electric field in the system. Different terms in the series are referred to as the solution of the corresponding order. We have presented analytical solutions of the relevant quantities up to the third order. Through our solutions we have demonstrated that in the presence of the magnetic field the pure upper-hybrid mode does not grow in time until the second order solutions. Our analysis also show that the slow component of the Lorentz force balances the direct effect of the ponderomotive forces, therefore we do not see any fast secular terms $\left(\sim t\right.$ or $\left.\sim t^{2}\right)$ in the second order solution. However, self-consistently generated cyclotron mode along with indirect effect of the ponderomotive forces introduce spatial dependency in the frequency of the system which triggers the phase mixing nonlinearly.

Using the similarities between an electron and a positron we also made a connection between the symmetry of the dynamical equations to that found in the nonlinear upper-hybrid mode. An interesting consequence of the symmetry is that the sum of the density fluctuations vanish in the third order, although third order solutions in general grow with time as $t \cos \omega_{h} t$ where $\omega_{h}$ is the upper-hybrid frequency. Through a 1D PIC simulation we have then confirmed our analytical results and have analyzed the evolution of the primary mode of oscillations in detail. We have estimated the phase-mixing time, after which the primary mode decays to " $1 / e$ " of initial amplitude, using our simulations. Our analysis show that the phase-mixing time $\propto \delta^{-0.83}$ whereas its dependence on the magnetic field does not follow a simple power law. However, our analysis clearly demonstrate that the phase mixing of electrostatic oscillations in a magnetized e-p plasma is significantly delayed compared to that in an unmagnetized e-p plasma. In addition, our above findings illustrate striking contrasts between the pure upper-hybrid oscillations studied in this paper and the mixed upper-hybrid mode studied in the Ref. 32 .

Furthermore, we have presented a generalization of the above mentioned symmetry identified in the nonlinear solution of the pure upper-hybrid mode to a traveling wave propagating at the upper-hybrid frequency. With 
the help of this symmetry we have constructed an unique nonlinear solution in a magnetized e-p plasma which does not show any signature of phase mixing. As a result the wave continues to propagate at the upper-hybrid frequency without damping.

In our analysis we have neglected the thermal effects. These effects are negligible in many situations of interests where the frequency $\omega>>k v_{t h}\left[3\right.$, here $v_{t h}$ stands for the thermal velocity and $k$ is the wavenumber. However, for more general situations the thermal and viscous effects should be considered. Investigations along these directions are in progress and would be reported else where. Examining the role of the relativistic effects on the upper-hybrid oscillations in an e-p plasma, which has direct applications in the wake-field acceleration ex- periments, could also be a possible future direction of our work. We conclude by noting that our results are relevant for experiments as in real situations it is natural to excite a single frequency coherent mode that is not accompanied by any specific zero-frequency mode and hence the results are important to understand and design probable controlled experiments.

\section{Appendix: Third order solutions}

From the set of Eqs. (48)-(54) we construct a second order partial differential equation for $n_{d}^{(3)}$ similar to the one in Sect. II A for $n_{d}^{(1)}$ and obtain the solution as,

$$
\begin{aligned}
& n_{d}^{(3)}=\frac{\delta^{3} n_{0} \cos k x}{64 \omega_{c}^{3}\left[\omega_{c}^{2}-4 \omega_{h}^{2}\right)^{2}}\left\{64 \omega_{c}^{4} \omega_{h}^{3}[2-3 \cos (2 k x)] \cos \left(\omega_{h} t\right) \sin \left(\omega_{c} t\right)-128 \omega_{c}^{2} \omega_{h}^{5}[2-3 \cos (2 k x)] \cos \left(\omega_{h} t\right) \sin \left(\omega_{c} t\right)\right. \\
& +64 \omega_{h}^{7}[2-3 \cos (2 k x)] \cos \left(\omega_{h} t\right) \sin \left(\omega_{c} t\right)+4 \omega_{c}^{3} \omega_{h}^{4}\left[34 \sin \left(\omega_{c} t-\omega_{h} t\right)-93 \sin \left(\omega_{h} t\right)+138 \cos (2 k x) \sin \left(\omega_{h} t\right)\right. \\
& +132 \cos (2 k x) \cos \left(\omega_{c} t\right) \sin \left(\omega_{h} t\right)-45 \cos (2 k x) \cos \left(2 \omega_{h} t\right) \sin \left(\omega_{h} t\right)+13 \sin \left(3 \omega_{h} t\right)-34 \sin \left(\omega_{c} t+\omega_{h} t\right) \\
& \left.+18 \omega_{h}[2-3 \cos (2 k x)] t \cos \left(\omega_{h} t\right)\right]-32 \omega_{c} \omega_{h}^{6}\left[4\left[3-2 \cos \left(\omega_{h} t\right)\right] \sin \left(\omega_{h} t\right)+3\left[7+5 \cos \left(\omega_{h} t\right)\right] \sin \left(\omega_{h} t\right) \cos (2 k x)\right. \\
& \left.+2 \omega_{h}[2-3 \cos (2 k x)] t \cos \left(\omega_{h} t\right)\right]+\omega_{c}^{7}\left[-[4-9 \cos (2 k x)]\left[2+\cos \left(2 \omega_{h} t\right)\right] \sin \left(\omega_{h} t\right)\right. \\
& \left.-2 \omega_{h}[2-3 \cos (2 k x)] t \cos \left(\omega_{h} t\right)\right]+\omega_{c}^{5} \omega_{h}^{2}\left[-8 \sin \left(\omega_{c} t-\omega_{h} t\right)+129 \sin \left(\omega_{h} t\right)-222 \cos (2 k x) \sin \left(\omega_{h} t\right)\right. \\
& -48 \cos (2 k x) \cos \left(\omega_{h} t\right) \sin \left(\omega_{h} t\right)+9 \cos (2 k x) \cos \left(2 \omega_{h} t\right) \sin \left(\omega_{h} t\right)-5 \sin \left(3 \omega_{h} t\right)+8 \sin \left(\omega_{c} t+\omega_{h} t\right) \\
& \left.\left.-6 \omega_{h}[2-3 \cos (2 k x)] t \cos \left(\omega_{h} t\right)\right]\right\} \\
& E_{x}^{(3)}=\frac{\delta^{3}\left(m \omega_{p}^{2} / e\right) \sin k x}{128 k \omega_{c}^{3}\left[\omega_{c}^{2}-4 \omega_{h}^{2}\right)^{2}}\left\{\omega_{c}^{7}\left[3[2+3 \cos (2 k x)] \sin \left(\omega_{h} t\right)+[2+3 \cos (2 k x)] \sin \left(3 \omega_{h} t\right)\right]\right. \\
& +\omega_{c}^{7} \omega_{h} 4 \cos (2 k x) \cos \left(2 \omega_{h} t\right)+2 \omega_{c}^{5} \omega_{h}^{2}\left[8 \sin \left(\omega_{c} t-\omega_{h} t\right)-22 \sin \left(\omega_{h} t\right)-74 \cos (2 k x) \sin \left(\omega_{h} t\right)\right. \\
& \left.-16 \cos \left(\omega_{c} t\right) \sin \left(\omega_{h} t\right)+3 \cos \left(3 \omega_{h} t\right) \sin \left(\omega_{h} t\right)-2 \sin \left(3 \omega_{h} t\right)-8 \sin \left(\omega_{c} t+\omega_{h} t\right)\right] \\
& +4 \omega_{c}^{4} \omega_{h}^{3}\left[-32 \cos (2 k x) \cos \left(\omega_{h} t\right) \sin \left(\omega_{h} t\right)+3 \omega_{c} \cos (2 k x) t \cos \left(\omega_{h} t\right)\right]+8 \omega_{c}^{3} \omega_{h}^{4}\left[-10 \sin \left(\omega_{c} t-\omega_{h} t\right)\right. \\
& +14 \sin \left(\omega_{h} t\right)+46 \cos (2 k x) \sin \left(\omega_{h} t\right)+44 \cos (2 k x) \cos \left(\omega_{h} t\right) \sin \left(\omega_{h} t\right)-15 \cos (2 k x) \cos \left(2 \omega_{h} t\right) \sin \left(\omega_{h} t\right) \\
& \left.-2 \sin \left(3 \omega_{h} t\right)+10 \sin \left(\omega_{c} t+\omega_{h} t\right)\right]+16 \omega_{c}^{2} \omega_{h}^{5}\left[16 \cos (2 k x) \cos \left(\omega_{h} t\right) \sin \left(\omega_{c} t\right)-9 \omega_{c} \cos (2 k x) t \cos \left(\omega_{h} t\right)\right] \\
& -64 \omega_{c} \omega_{h}^{6}\left[2 \sin \left(\omega_{h} t\right)+7 \cos (2 k x) \sin \left(\omega_{h} t\right)+2 \cos \left(\omega_{c} t\right) \sin \left(\omega_{h} t\right)+5 \cos (2 k x) \cos \left(\omega_{c} t\right) \sin \left(\omega_{h} t\right)\right] \\
& \left.-128 \omega_{h}^{7}\left[\cos (2 k x) \cos \left(\omega_{h} t\right) \sin \left(\omega_{c} t\right)-\omega_{c} \cos (2 k x) t \cos \left(\omega_{h} t\right)\right]\right\}
\end{aligned}
$$

[1] J. M. Dawson, Physical Review 113, 383 (1959).

[2] R. Davidson and P. Schram, Nuclear Fusion 8, 183 
(1968)

[3] R. Davidson, Academic, New York (1972).

[4] L. Stenflo and O. Gradov, Physical Review E 58, 8044 (1998).

[5] G. Brodin and L. Stenflo, Physics Letters A 378, 1632 (2014).

[6] G. Brodin and L. Stenflo, Physics of Plasmas 21, 122301 (2014).

[7] G. Brodin and L. Stenflo, Physics Letters A 381, 1033 (2017).

[8] J. Faure, Y. Glinec, A. Pukhov, S. Kiselev, S. Gordienko, E. Lefebvre, J.-P. Rousseau, F. Burgy, and V. Malka, Nature 431, 541 (2004).

[9] A. Caldwell, K. Lotov, A. Pukhov, and F. Simon, Nature Physics 5, 363 (2009).

[10] M. Litos, E. Adli, W. An, C. Clarke, C. Clayton, S. Corde, J. Delahaye, R. England, A. Fisher, J. Frederico, et al., Nature 515, 92 (2014).

[11] A. I. Akhiezer and R. Polovin, Soviet Phys. JETP 3 (1956).

[12] P. S. Verma, S. Sengupta, and P. Kaw, Physical Review E 86, 016410 (2012).

[13] P. K. Kaw, A. Lin, and J. Dawson, The Physics of Fluids 16, 1967 (1973).

[14] E. Infeld, G. Rowlands, and S. Torven, Physical Review Letters 62, 2269 (1989).

[15] C. Nappi, A. Forlani, and R. Fedele, Physica scripta 43, 301 (1991).

[16] E. Infeld and G. Rowlands, Physical Review Letters 62, 1122 (1989).

[17] S. Sengupta, V. Saxena, P. K. Kaw, A. Sen, and A. Das, Physical Review E 79, 026404 (2009).
[18] P. S. Verma, S. Sengupta, and P. Kaw, Physical Review Letters 108, 125005 (2012).

[19] C. Maity, N. Chakrabarti, and S. Sengupta, Physical Review E 86, 016408 (2012).

[20] S. S. Gupta and P. K. Kaw, Physical Review Letters 82, 1867 (1999).

[21] P. S. Verma, Physics of Plasmas 18, 122111 (2011).

[22] E. Saberian and A. Esfandyari-Kalejahi, Physical Review E 87, 053112 (2013).

[23] P. Helander, Physical Review Letters 113, 135003 (2014).

[24] M. R. Edwards, N. J. Fisch, and J. M. Mikhailova, Physical Review Letters 116, 015004 (2016).

[25] Y.-M. Wang, M. Yu, L. Stenflo, and A. Karimov, Chinese Physics Letters 33, 085205 (2016).

[26] H. Chen, S. C. Wilks, J. D. Bonlie, E. P. Liang, J. Myatt, D. F. Price, D. D. Meyerhofer, and P. Beiersdorfer, Physical Review Letters 102, 105001 (2009).

[27] P. Sturrock, The Astrophysical Journal 164, 529 (1971).

[28] F. C. Michel, Reviews of Modern Physics 54, 1 (1982).

[29] M. C. Begelman, R. D. Blandford, and M. J. Rees, Reviews of Modern Physics 56, 255 (1984).

[30] C. Maity, A. Sarkar, P. K. Shukla, and N. Chakrabarti, Physical Review Letters 110, 215002 (2013).

[31] M. Karmakar, C. Maity, and N. Chakrabarti, Physics of Plasmas 23, 064503 (2016).

[32] C. Maity, N. Chakrabarti, and S. Sengupta, Physics of Plasmas 20, 082302 (2013).

[33] G. Stewart, Journal of plasma physics 50, 521 (1993).

[34] C. Maity, Physics of Plasmas 21, 072317 (2014).

[35] P. S. Verma, Physics of Plasmas 23, 122125 (2016).

[36] C. K. Birdsall and A. B. Langdon, Plasma physics via computer simulation (CRC Press, 2004). 